\title{
Caso de estudio sobre la innovación social en cuatro ciudades de Colombia: Bogotá, Buenaventura, Cali y Cartagena
}

\author{
Rosa Alexandra Chaparro-Guevara*, Edwin Leonardo Méndez-Ortíz ${ }^{* *}$
}

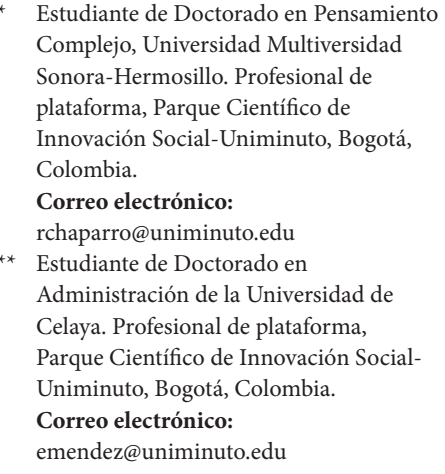

Recibido: 25 de julio del 2015

Aprobado: 9 de noviembre del 2015

Cómo citar este artículo: Chaparro-Guevara, R. A. y Méndez-Ortíz, E. L. (2016). Caso de estudio sobre la innovación social en cuatro ciudades del país: Bogotá, Buenaventura, Cali y Cartagena. Cooperativismo \& Desarrollo, 24(108), 55-70. doi: http://dx.doi. org/10.16925/co.v24i108.1260

\section{Resumen}

Introducción: este artículo evidencia un trabajo realizado en cuatro ciudades de Colombia con uno de los premios de mayor trayectoria en el ámbito social colombiano: Premio Cívico de la Fundación Corona. Metodología: se realizó un estudio mixto con enfoque descriptivo, hallando como resultado seis variables independientes que explican el comportamiento de la innovación: experiencia, número de integrantes de la organización, participación de la comunidad en la iniciativa, si las iniciativas han sido finalistas del premio en otras ocasiones, monto de financiación utilizado y si la iniciativa posee personería jurídica. Resultados: en relación con la fase cualitativa, se hallaron seis elementos significativos dentro de la misión de las organizaciones relacionadas con proyectos de innovación social: mejora de la calidad de vida de los participantes y la comunidad, construcción de paz, promoción de la cultura, espacios de participación, aprovechamiento del tiempo libre y fortalecimiento del tejido social. Conclusiones: este caso de estudio permite identificar, a la luz de la innovación social, las principales características de las organizaciones de base del ámbito de la acción social, en Bogotá, Buenaventura, Cali y Cartagena.

Palabras clave: comunidad, iniciativas, innovación social, modelo logit, organizaciones, premio. 


\title{
Case Study on Social Innovation in Four Cities in Colombia: Bogotá, Buenaventura, Cali and Cartagena
}

\begin{abstract}
Introduction: This article shows a work performed in four cities in Colombia with one of the longest running awards in the Colombian social sphere: Fundación Corona's Civic Award. Methodology: A mixed study with a descriptive approach was conducted resulting in six independent variables that explain the behavior of innovation: experience, number of members in the organization, community participation in the initiative, if the initiatives have been nominees for the award at other times, amount of funding used, and whether the initiative has legal status. Results: In relation to the qualitative phase, six significant elements were found in the mission of organizations related to social innovation projects: improved quality of life of participants and the community, peace-building, promotion of culture, opportunities for participation, use of leisure time, and strengthening of social fabric. Conclusions: This study case allows to identify, in the light of social innovation, the main features of grassroots organizations in the field of social action, in Bogotá, Buenaventura, Cali and Cartagena.
\end{abstract}

Keywords: community, initiatives, social innovation, logit model, organizations, award.

\section{Caso de estudo sobre a inovação social em quatro cidades da Colômbia: Bogotá, Buenaventura, Cali e Cartagena}

\section{Resumo}

Introdução: este artigo evidencia um trabalho realizado em quatro cidades da Colômbia com um dos prêmios de maior trajetória no âmbito social colombiano: "Premio Cívico" da Fundación Corona. Metodologia: realizou-se um estudo misto com enfoque descritivo, encontrando como resultado seis variáveis independentes que explicam o comportamento da inovação: experiência, número de integrantes da organização, participação da comunidade na iniciativa, se as iniciativas têm sido finalistas do prêmio em outras ocasiões, montante de financiamento utilizado e se a iniciativa possui natureza jurídica. Resultados: quanto à fase qualitativa, constataram-se seis elementos significativos dentro da missão das organizações relacionadas com projetos de inovação social: melhoria da qualidade de vida dos participantes e da comunidade, construção da paz, promoção da cultura, espaços de participação, aproveitamento do tempo livre e fortalecimento do tecido social. Conclusões: este caso de estudo permite identificar, à luz da inovação social, as principais características das organizações de base do âmbito da ação social em Bogotá, Buenaventura, Cali e Cartagena.

Palavras-chave: comunidade, iniciativas, inovação social, modelo logit, organizações, prêmio. 


\section{Introducción}

El Observatorio de Innovación Social del Parque Científico de Innovación Social-PCIs de Uniminuto adelantó, durante los meses de septiembre y octubre del 2014, una serie de visitas a iniciativas sociales, planteadas dentro del convenio marco de cooperación entre entidades que promueven el fortalecimiento de iniciativas sociales presentadas al Premio Cívico de la Fundación Corona, teniendo como objetivos facilitar la articulación de las iniciativas comunitarias y/o colectivas socialmente innovadoras y la construcción de lo público, mediante un ciclo constituido por los procesos de convocatoria, identificación, reconocimiento, fortalecimiento y sistematización de las experiencias en Bogotá, Buenaventura, Cali y Cartagena.

El proceso de sistematización se desarrolló con la participación de profesionales del Observatorio de Innovación Social ${ }^{1}$, en compañía del equipo líder de Premio Cívico de la Fundación Corona², los cuales a través de entrevistas semiestructuradas se acercaron a las iniciativas con el fin de reconocer su proceso de conformación y aportes a la comunidad. Así mismo se analizó la base de datos entregada por Premio Cívico para la caracterización de las organizaciones que se inscribieron en el 2013.

Se hizo un ejercicio de construcción colectiva y de reflexión a partir de las vivencias personales de quienes conformaban las iniciativas, reconociendo los aciertos, desaciertos y principales aprendizajes que contribuyeron a la mejora de las condiciones de vida de los habitantes de las zonas de influencia de los proyectos que participaron en la versión del premio para el 2013.

\section{Antecedentes}

En Bogotá, en 1998, se realizó el primer Premio Cívico. La intención con la cual se creó se basó en la construcción de tejido social a partir de propuestas de iniciativas innovadoras, lo cual convirtió a la innovación social en el eje del premio; de este modo se buscó que las propuestas presentaran alternativas a las problemáticas sociales detectadas en sus entornos. Posteriores ediciones del premio se realizaron en Cali (2006), Cartagena (2007) y Buenaventura (2013). Las iniciativas propusieron soluciones a situaciones problémicas especialmente en temas de cultura, infancia,

1 Carlos Vásquez, Paula Alejandra López, Edwin Méndez, Daniel Trujillo, Nicolás Gaitán.

2 Paola Avendaño, Francy Barbosa. infraestructura, medio ambiente, recreación y atención a población en estado de vulnerabilidad.

Los componentes de juicio para otorgar el premio giraron alrededor de la promoción de iniciativas innovadoras y colectivas, la gestión del conocimiento y la capacidad de incidir en escenarios públicos (Premio Cívico, 2013).

\section{Fundamentos Teóricos}

\section{Innovación Social}

Para comenzar este apasionante recorrido por la innovación social, es importante que se reconozcan los principales aportes que desde diferentes enfoques se desarrollan alrededor de este concepto. Bouchard (2007), en su escrito de economía social, reconoce la innovación social como un proceso colectivo en el cual las innovaciones de otro orden, como las científicas y las tecnológicas, tienen implícitas las innovaciones sociales, ya que las entiende como las nuevas formas en las que se organiza el trabajo. Esta autora reconoce que las innovaciones tecnológicas tienen un componente de innovación social. Asimismo, para Alter (2002), la innovación social y el emprendimiento social, se consideran como una serie de acciones que se desarrollan en la vida cotidiana con el fin de generar confianza, respeto y transparencia, es decir que para los anteriores autores la innovación social tiene un alto componente de transversalidad en la vida cotidiana.

Para que se desarrolle la innovación social, es necesario que se efectúen rupturas de formas tradicionales que, como lo menciona Hollingsworth (2000), se encuentran desde las asociaciones académicas y las diferentes disciplinas, de manera que se convierten en una característica de la universidad actual y esto dificulta el desarrollo de las innovaciones. Es decir, que para promover la innovación, se hace necesaria la articulación de diversos campos del conocimiento, así como de las diferentes disciplinas, según el alcance de los objetivos propuestos.

Teniendo en cuenta los elementos planteados anteriormente, Uniminuto se interesa por articular el modelo de investigación, desarrollo e innovación, integrando funciones sustantivas de educación superior (docencia, investigación y extensión) que permiten mejorar la productividad y la competitividad, basándose en la generación y apropiación social del conocimiento. A continuación, se presentan algunos de los elementos que identifican la innovación social como lo plantea Rocha (2011). 


\section{Ideas + Propuestas}

La innovación social surge de ideas o propuestas innovadoras, que pueden ser desarrolladas por cualquiera de los actores de la sociedad; si es por cualquier actor de la sociedad, existe por tanto un reto que se constituye en identificar estas ideas, promover la generación de las mismas y generar un espacio en el cual ellas broten. Soluciones a pequeña escala: las ideas innovadoras se convierten en prototipos o pequeños laboratorios de experimentación en donde las soluciones implementadas pueden ser sometidas a prueba en condiciones reales.

\section{Sostenibilidad}

Se revisan las condiciones de sostenibilidad de estas soluciones a pequeña escala, a fin de determinar si al remover el impulso inicial de capital o trabajo, la solución implementada es sostenible y durable en el tiempo.

\section{Escalamiento + Difusión}

Las soluciones a pequeña escala, después de haber probado su sostenibilidad, deben pasar a un proceso de escalamiento de las soluciones, que implica la réplica de esta, además de construir las condiciones adecuadas de réplica para promover su escalamiento en otros contextos.

\section{Nuevas propuestas}

El desarrollo del escalamiento y la difusión de soluciones innovadoras puede ser a su vez una nueva fuente de ideas y de propuestas que genere un círculo virtuoso de desarrollo de soluciones. De igual modo, se muestra la forma como se genera la innovación social, desde los actores, las problemáticas, las áreas temáticas, los aliados, los gestores y la experimentación social. Para el presente estudio, se entiende por innovación social la búsqueda e implementación de soluciones novedosas, eficientes, participativas y sustentables a los problemas que limitan el logro de mejores condiciones de vida en una comunidad.

\section{Sistematización de experiencias}

La sistematización de experiencias para América Latina toma importancia en la década de los ochenta, gracias al interés por documentar los procesos, aprendizajes y participación de las comunidades:
"La sistematización se conceptualiza como una forma de generación de conocimientos adecuada a las condiciones de trabajo y capacidades particulares de quienes están involucrados cotidianamente en la ejecución de las acciones y que son, prácticas" (Francke y Morgan, 1995, p. 5).

Se convierte en una alternativa a las formas de evaluar los procesos, ya que, en muchas de las oportunidades, se da cuenta del cumplimiento de objetivos del proyecto, pero no se profundiza en riquezas de las experiencias vividas, ya que la sistematización busca la participación de los actores desde sus propias experiencias, sus capacidades y la posibilidad de conocerse, reconocerse y generar redes y alianzas.

Retomando la propuesta efectuada por Holliday, los tiempos de sistematización para el presente proceso de Premio Cívico giran alrededor de cinco elementos principales, los cuales se describen a continuación:

Punto de partida: en esta fase se deben identificar las características propias del proceso, si hay registros, las condiciones bajo las cuales se da inicio al proceso de sistematización.|

Preguntas iniciales: en esta fase se redactan las preguntas orientadoras para el proceso de sistematización.

Recuperación del proceso vivido: aquí se reconstruye la historia de las iniciativas a partir de las mismas preguntas orientadoras y de la narrativa que realizan los participantes, así como la participación de los talleres de construcción de línea de tiempo.

Reflexión de fondo: se analiza y se recupera la información, previa clasificación de la misma.

Puntos de llegada: se formulan las conclusiones, se redactan los aprendizajes y se divulgan los resultados.

La propuesta de sistematización se soporta en la orientación teórico-práctica propuesta por Holliday. A continuación, se identifican las preguntas iniciales bajo las cuales girará el proceso de sistematización: ¿para qué se quiere sistematizar?, ¿qué se quiere sistematizar, ¿qué tipo de experiencias?

\section{Organizaciones sociales y comunitarias}

Las organizaciones comunitarias se derivan, como lo plantea Escobar Delgado (2010, citando a Weber), a partir de las relaciones entre los sujetos que identifican una situación común para alcanzar propósitos comunes, es decir, que un patrón que caracteriza a este tipo de organizaciones tiene que ver con la forma en la que se organizan, en tanto se busca alcanzar objetivos y 
metas, este no es un concepto nuevo, por el contrario desde la década de los sesenta se habla de él.

Autores como Etzioni (1975) y Scott (1963) coinciden con elementos planteados por Weber, ya que las personas se organizan para alcanzar objetivos y metas. Estas se entienden como colectivos que se constituyen a la luz de unas metas comunes. Se consideran las organizaciones como los espacios en los cuales se puede aportar a la prosperidad, la paz y la justicia social. El trabajo en las organizaciones por parte de los sujetos les permite integrarse y dar respuesta a sus necesidades.

Para Richard (1983), una organización se convierte en un colectivo que se identifica con una serie de normas, autoridades, comunicaciones, se desarrolla en un ambiente buscando alcanzar objetivos y metas para sí mismas, sus trabajadores y la sociedad en general.

\section{Especificación del modelo Logit Binomial}

La determinación que una iniciativa sea innovación social es la variable dependiente del modelo, es discreta, ya que solo puede tomar valores enteros, y expresa varias alternativas ordinales mutuamente excluyentes, en este caso, la regresión lineal no es adecuada para explicar las particularidades de esta variable endógena dado que presenta tres problemas: primero, acotamiento: debido a que las predicciones de la probabilidad pueden no estar dentro del rango de $(0,1)$. Segundo, discretitud: la variable dependiente es una variable discreta y el modelo de regresión lineal es para variables continuas; y tercero, incumple el supuesto de normalidad en los errores, por lo cual la varianza de los errores no es constante, es decir se presenta heterocedasticidad. Por estas tres razones, en los modelos de variable dependiente discreta no se utiliza el método de mínimos cuadrados.

Los modelos de variable dependiente discreta resuelven los problemas de acotamiento, discretitud y no normalidad mediante el método de máxima verosimilitud, el cual consiste en maximizar la función de verosimilitud. Este método tiene la ventaja de que no requiere del supuesto de normalidad en los errores, sin embargo, si el error se distribuye normal los estimadores de máxima verosimilitud coinciden con los obtenidos por el método de mínimos cuadrados (Davidson y MacKinnon, 2004).

$$
\begin{gathered}
y_{i}=\text { Noesinnovaciónsociallainiciativay }_{i}=0 \\
\text { con probabilidad } \mathrm{P}
\end{gathered}
$$

$$
\begin{gathered}
y_{i}=\text { Esinnovaciónsociallainiciativay }_{i}=1 \\
\text { con probabilidad } 1-\mathrm{p}
\end{gathered}
$$

El número de umbrales equivale al número de alternativas menos 1 , este límite $\gamma_{1}$ (desconocido $a$ priori y estimado en el modelo) determina los valores de $y_{i}^{o}$, y se traducen en las dos alternativas posibles de $y_{i}$. Para que el modelo cumpla con la condición de orden $\gamma_{0}$ debe ser menor que $\gamma_{1}$. En todos los casos, la probabilidad de cada una de las dos alternativas $\left(y_{i}=0, y_{i}=1\right)$ debe ser positiva y estan definidas por:

Para que no sea una iniciativa innovadora socialmente (0):

$$
\begin{aligned}
\operatorname{Pr}\left(y_{i}=0\right)= & \operatorname{Pr}\left(y_{i}^{o}<\gamma_{1}\right) \\
& =\operatorname{Pr}\left(\boldsymbol{X}_{i} \beta+u_{i}<\gamma_{1}\right) \\
& =\operatorname{Pr}\left(u_{i}<\gamma_{1}-\boldsymbol{X}_{i} \beta\right) \\
& =\Phi\left(\gamma_{1}-\boldsymbol{X}_{i} \beta\right)
\end{aligned}
$$

Para ser una iniciativa innovadora socialmente (1):

$$
\begin{aligned}
\operatorname{Pr}\left(y_{i}=1\right)=1 & -\operatorname{Pr}\left(y_{i}=0\right) \\
= & 1-\Phi\left(\gamma_{1}-\boldsymbol{X}_{i} \beta\right)
\end{aligned}
$$

En donde, es la función de distribución acumulada de los errores de la cual depende la especificación del modelo, si es un logit ordenado se modela como una función logística.

$$
\Phi=\frac{\mathrm{e}^{\mathrm{X}_{\mathrm{i}} \beta}}{\left(1+\mathrm{e}^{\mathrm{X}_{\mathrm{i}} \beta}\right)}
$$

\section{Metodología}

La metodología utilizada durante este proceso tuvo un enfoque mixto. En el enfoque cualitativo, las herramientas utilizadas para el levantamiento de la información fueron las entrevistas semiestructuradas, buscando la reconstrucción propia de cada organización y de la iniciativa. El principio que fundamentó está técnica de levantamiento de información radica en la necesidad de contactar de forma directa a los sujetos y escenarios propios que fueron finalistas de Premio Cívico. 
La construcción de los antecedentes históricos tanto de la iniciativa como de la organización evidencian la forma en la que se organiza la comunidad para mostrar soluciones alternativas a una serie de problemáticas que se presentan en su entorno. El software utilizado en el apartado cualitativo fue el Atlas.ti y las nubes de palabras en Tagxedo.

Con relación al enfoque cuantitativo, se trabajó con la base entregada por el premio y a la luz de logit ordenado separando cada ciudad analizada. El software utilizado para esta parte del estudio fue el Stata.

\section{Perfiles de las organizaciones}

En la figura 1, se presenta la distribución de las iniciativas según las divisiones administrativas de cada ciudad (localidades/comunas).
Como se puede apreciar en la figura 1, en Bogotá, las iniciativas se encuentran distribuidas en 17 de las 20 localidades. Los porcentajes más grandes están radicados en Ciudad Bolívar y Chapinero; en Ciudad Bolívar, la principal razón es la presencia de población con alto grado de vulnerabilidad, mientras que, para el caso de Chapinero, aunque esta localidad no sea el área de desarrollo de la iniciativas, es una zona céntrica para la instalación de las sedes administrativas de los diferentes proyectos.

Por otro lado, se evidencia la ausencia de Tunjuelito, Teusaquillo y Sumapaz. La ausencia de la localidad de Sumapaz es acorde con la poca cantidad de proyectos de carácter rural desarrollados en la ciudad, territorio con gran potencialidad siendo la localidad con mayor extensión de todo Bogotá.

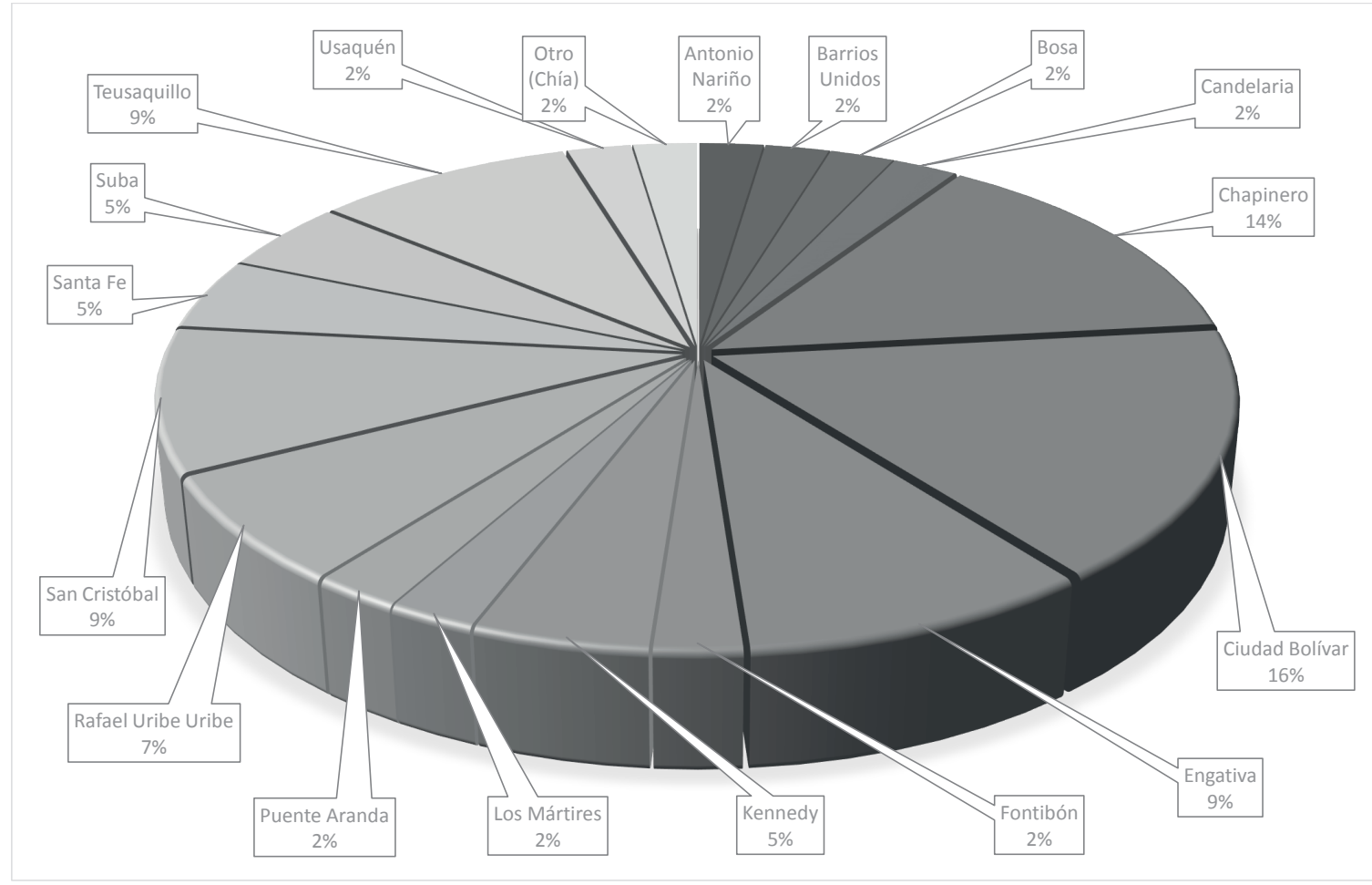

Figura 1. Ubicación de organizaciones en Bogotá.

En cuanto a Cali, como se observa en la figura 2, el mayor número de iniciativas se concentra en las comunas 13, 14 y 21, las cuales, según datos del Observatorio Social de Cali (2014), evidencian problemáticas de seguridad y violencia; esta es una de las razones por las cuales algunas de las iniciativas trabajan especialmente en temas de derechos humanos como se puede apreciar más adelante en el presente documento.

En cuanto a Buenaventura (figura 4), el mayor número de iniciativas se concentra en las comunas 8 , 11 y 12 . Se caracterizan por dificultades de empleo y de seguridad (Alcaldía Buenaventura, 2014). 


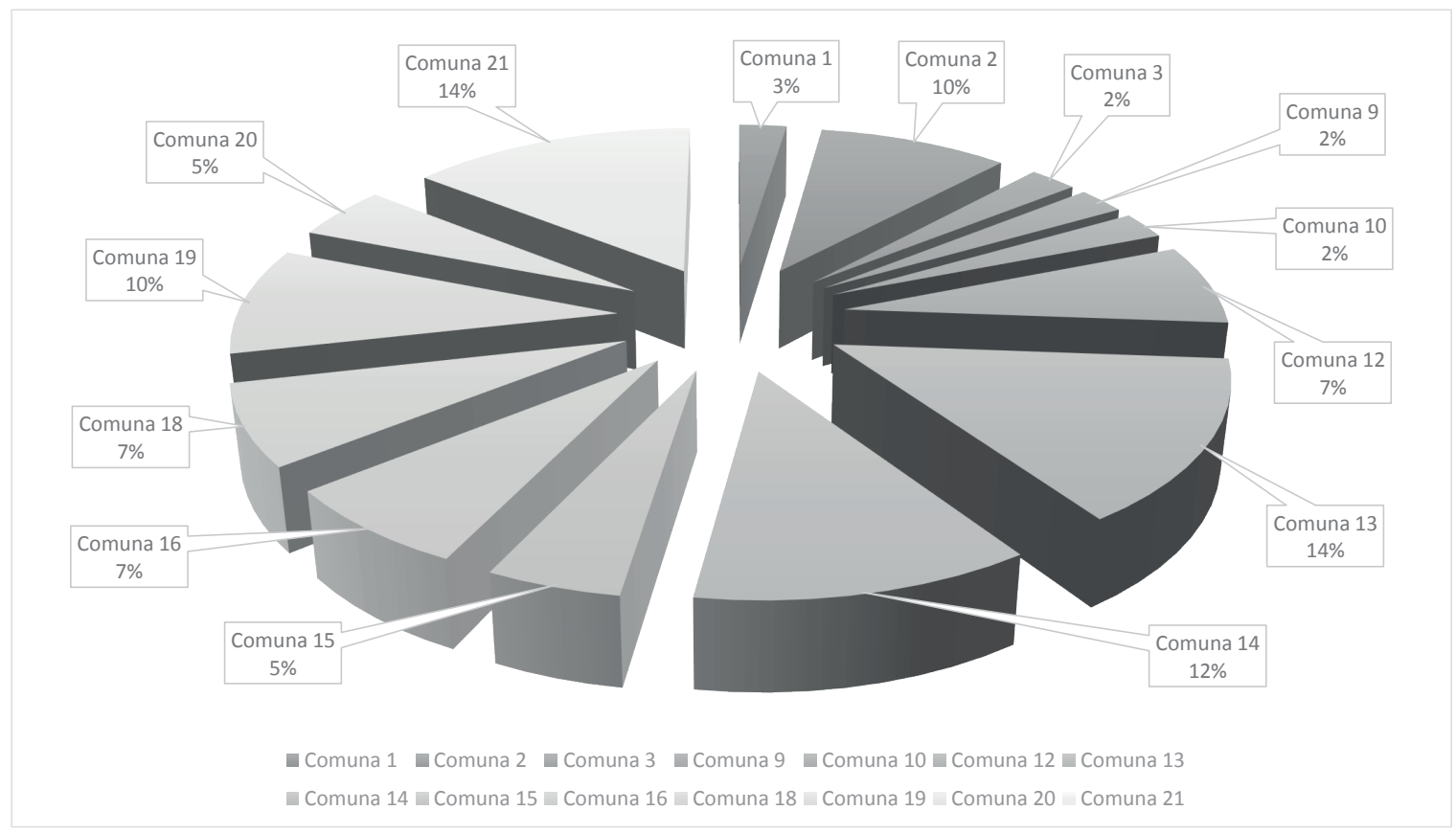

Figura 2. Ubicación de organizaciones en Cali.

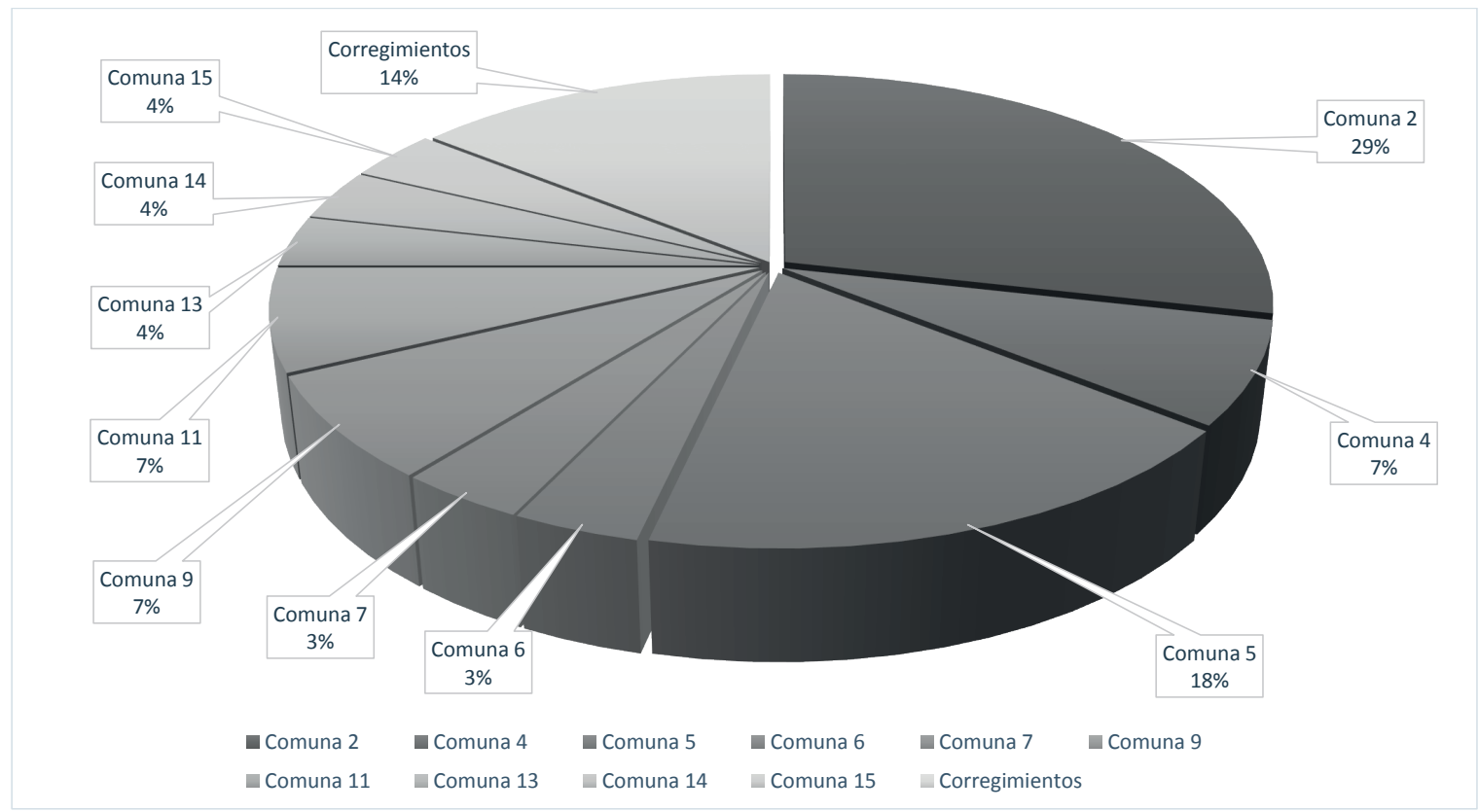

Figura 3. Ubicación de organizaciones en Cartagena. 


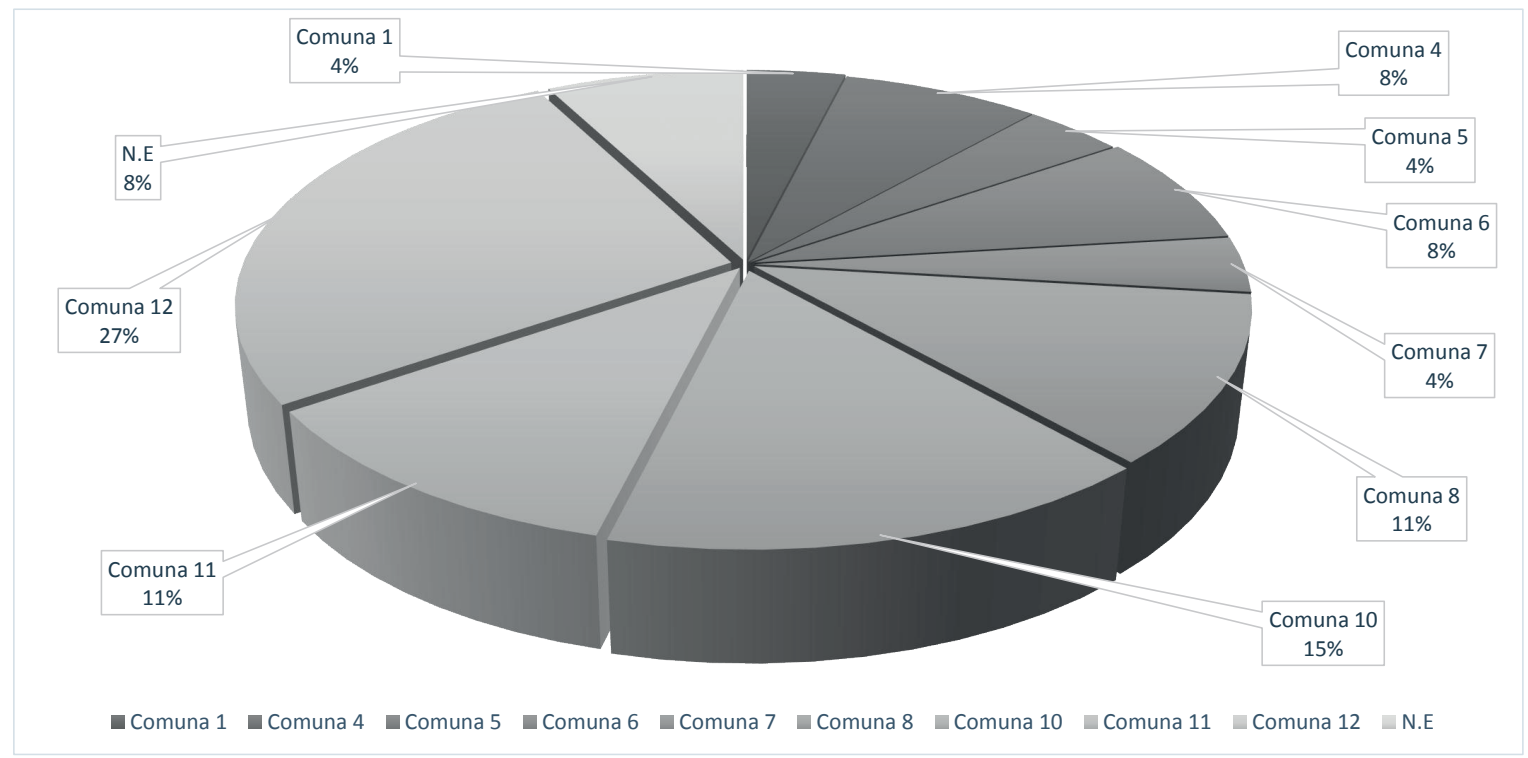

Figura 4. Ubicación de organizaciones en Buenaventura.

\section{Descripción de variables para el estudio cuantitativo}

La variable dependiente utilizada es innovación, la cual tiene un carácter dicotómico, es decir, toma los dos valores posibles de 0 y 1 , dependiendo de si fue o no finalista en premio cívico, en donde es 0 si no fue finalista y 1 si fue finalista y tiene una media de 0,27. Según esta categorización, se corrió un modelo logit ordenado y se encontraron los siguientes resultados según la tabla 1.

Tabla 1

Variable de Innovación

\begin{tabular}{cccccc}
\hline Variable & Obs & Mean & Std. Dev. & Min & Max \\
Innovación & 135 & 0,2740741 & 0,4477078 & 0 & 1 \\
\hline
\end{tabular}

Nota. Salidas del software Stata versión 10. Elaboración propia.

Las variables independientes utilizadas para explicar el comportamiento de la innovación, de acuerdo con las posibilidades de los datos levantados por el premio fueron: experiencia, número de integrantes de la organización, participación de la comunidad en la iniciativa, si las iniciativas han sido finalistas del premio en otras ocasiones, monto de financiación utilizado y si la iniciativa posee personería jurídica. En la tabla 2, se explica cada una de estas variables.
Tabla 2

Experiencia de las organizaciones

\begin{tabular}{cccccc}
\hline Variable & Obs & Mean & Std. Dev. & Min & Max \\
Experiencia & 135 & 6,333333 & 5,567764 & 2 & 43 \\
\hline
\end{tabular}

Nota. Salidas del software Stata versión 10. Elaboración propia.

La variable experiencia se entiende como el tiempo de funcionamiento de la iniciativa hasta el momento actual, medida en años. Se espera que un mayor tiempo de experiencia pueda mejorar las condiciones para que una iniciativa sea innovadora socialmente o viceversa. A partir de la descripción estadística se obtiene que el tiempo promedio de funcionamiento de las 135 iniciativas observadas es de 6,3 años, con una desviación estándar de 5,6 años, así mismo se observa que la iniciativa con menor tiempo de funcionamiento encontrada tiene 2 años y la que mayor periodo de funcionamiento tiene es de 43 años.

Tabla 3

Integrantes de las organizaciones

\begin{tabular}{cccccc}
\hline Variable & Obs & Mean & Std. Dev. & Min & Max \\
Integrantes & 135 & 84,41481 & 315,1143 & 2 & 2500 \\
\hline
\end{tabular}

Nota. Elaboración propia. 
El número de integrantes de la iniciativa, como se puede apreciar en la tabla 3, fue la segunda variable dependiente utilizada para explicar la innovación social, esta tiene como características más importantes que en promedio hay 84 personas que conforman las organizaciones que desarrollan la iniciativa, con un valor mínimo de 2 integrantes y un valor máximo de 2500 integrantes. Posee una desviación estándar de 315. Como conclusión del análisis de esta variable, puede decirse que organizaciones con mayores integrantes poseen mejores condiciones para fortalecer procesos encaminados a innovación social, porque pueden tener mayores posibilidades de especialización del trabajo.

La siguiente variable explicativa se denomina "Participantes". Se refiere al número de beneficiarios de cada iniciativa y se espera que mayor número de ellos las acerquen a la innovación social, por temas de cobertura y de reconocimiento, entre otros.

De las 135 iniciativas presentadas a Premio Cívico estas cuentan con 130 beneficiarios en promedio, presentándose un valor máximo de 2500, un mínimo de 0 y una desviación estándar de 293. Estos datos se pueden observar en la tabla 4 .

Tabla 4

Participantes de las organizaciones

\begin{tabular}{cccccc}
\hline Variable & Obs & Mean & Std. Dev. & Min & Max \\
participantes & 135 & 130,237 & 293,0362 & 0 & 2500 \\
\hline
\end{tabular}

Nota. Elaboración propia.

La próxima variable explicativa, como se puede apreciar en la tabla 5, es de carácter "Dicotómico", a partir de la pregunta sobre si ha sido finalista de Premio Cívico en otras oportunidades, ante la que se presentan dos posibles resultados, en primera instancia, si lo ha sido toma el valor de 1 , en caso contrario, toma el valor de 0 , lo que conlleva a plantear que iniciativas finalistas tienen mayores probabilidades de ser socialmente innovadoras que las que no lo han sido, este puede ser el resultado de procesos previos de fortalecimiento y acompañamiento, también de una curva de aprendizaje menos pronunciada que en una iniciativa que no ha tenido algún reconocimiento.

La quinta variable explicativa seleccionada es la de "Financiamiento", como se puede apreciar en la tabla 6, compuesta por los valores que tiene cada iniciativa para financiarse. Estos valores recaen en el músculo financiero que posee la iniciativa y en la posibilidad de que brinde mayores oportunidades de operar y de ser sostenible en el tiempo, por esto se espera que a mayor poder financiero, haya mayores oportunidades de ser innovadora socialmente, teniendo en cuenta la capacidad de mantenerse en el tiempo y realizar mayores actividades que potencien su desarrollo. Debido a los valores del financiamiento, se tomó la decisión de aplicar logaritmo natural a esta variable.

Tabla 5

Ha sido finalista de premio

\begin{tabular}{cccccc}
\hline Variable & Obs & Mean & Std. Dev. & Min & Max \\
Ha sido finalista & 135 & 0,1333333 & 0,3412007 & 0 & 1 \\
\hline
\end{tabular}

Nota. Elaboración propia.

Tabla 6

Financiamiento

\begin{tabular}{cccccc}
\hline Variable & Obs & Mean & Std. Dev. & Min & Max \\
Financiamiento & 135 & 14,90504 & 5,255194 & 0 & 20,83 \\
\hline
\end{tabular}

Nota. Elaboración propia.

Tabla 7

Personeria jurídica

\begin{tabular}{cccccc}
\hline Variable & Obs & Mean & Std. Dev. & Min & Max \\
Personería & 135 & 0,6740741 & 0,4704655 & 0 & 1 \\
\hline
\end{tabular}

Nota. Elaboración propia.

La última variable tiene que ver con un tema de legalidad, es decir, si las organizaciones poseen personería jurídica, lo cual también está asociado a un tema de fortalecimiento organizacional y formalidad para operar y realizar actividades. Se espera que organizaciones en un marco de legalidad tengan más opción de realizar innovaciones sociales.

\section{Resultados para la fase cuantitativa}

El modelo que se corrió es un logit ordenado que espera explicar el comportamiento de la innovación social (tabla 8), donde se aprecia que la única variable que mantiene una relación inversa con la innovación 
Tabla 8

Logit innovación, experiencia, integrantes, finalista, personería jurídica

Iterration 0:

Iterration 1:

$\log$ likelihood $=-79,281319$

Iterration 2:

log likelihood $=-75,511325$

Iterration 3 :

$\log$ likelihood $=-75,396686$

$\log$ likelihood $=-75,396259$
$\log$ likelihood $=-75,396259$

Ordered logistic regression

Log likelihood - - -75,396259

$\begin{array}{llr}\text { Number of obs } & - & -135 \\ \text { LR chi2(6) } & - & -7,77 \\ \text { Prob > chi2 } & - & -0,25554 \\ \text { Pseudo R2 } & - & -0,0490\end{array}$

\begin{tabular}{lcccccc}
\hline Innovación & Coef. & Std. Err. & $\mathbf{z}$ & $\mathbf{P}>|\mathbf{z}|$ & \multicolumn{2}{c}{ [95\% Cont. Interval] } \\
\hline Experiencia & $-0,021628$ & 0,0389615 & $-0,56$ & 0,579 & $-0,0979912$ & 0,0547352 \\
Integrantes & 0,0003774 & 0,000724 & 0,52 & 0,602 & $-0,0010415$ & 0,0017964 \\
Participantes & 0,0005761 & 0,0007937 & 0,73 & 0,468 & $-0,0009795$ & 0,0021317 \\
Ha sido finalista & 0,6921239 & 0,5384955 & 1,29 & 0,199 & $-0,3633078$ & 1,747556 \\
Financiamiento & 0,0561177 & 0,0486716 & 1,15 & 0,249 & $-0,0392769$ & 0,1515124 \\
Personería & 0,66359734 & 0,4659319 & 1,42 & 0,154 & $-0,2496123$ & 1,576807 \\
\hline \multicolumn{1}{c}{ /cut1 } & 2,388848 & 0,8863451 & & & 0,6516436 & 4,126053 \\
\hline
\end{tabular}

Nota. Elaboración propia.

social es la experiencia, es decir, a mayores años de experiencia menores resultados de innovación social se tienen, por otra parte las demás variables mantienen una relación positiva con el fenómeno de estudio, lo que concuerda con las hipótesis planteadas.

Adicionalmente, ninguna de las seis variables independientes es significativa individualmente, con un margen de confiabilidad del $95 \%$, siendo la más significativa el tener personería jurídica, continuando con el haber sido finalista del premio en otras ocasiones, seguida del financiamiento, la participación de los beneficiarios, la experiencia y el número de integrantes de la organización respectivamente.

El valor de r2 $(0,049)$ es muy bajo, lo que concluye que las variables explicativas solo dan cuenta del comportamiento de la variable dependiente en un $4,9 \%$, por tales razones el modelo no es concluyente, esto puede estar asociado a los datos recolectados y a las preguntas realizadas en la captura de la información, es decir, si las variables investigadas realmente ayudan a comprender el fenómeno de la innovación social.

Debido a los resultados anteriores, se procede a correr nuevamente el modelo excluyendo de este las variables menos significativas individualmente, las cuales son la experiencia y los integrantes de las organizaciones.

Esta nueva salida excluye las variables menos significativas individualmente, mostrando que las siguientes variables (participantes, si ha sido finalista de otros premios en otras ocasiones, financiamiento y personería jurídica) mantienen una relación positiva con la innovación social, pero ninguna es significativa al 95\% de confianza, adicionalmente, solo explican el comportamiento de la innovación social en un 4,57\%, por tanto, el modelo no es concluyente (tabla 9).

Finalmente, se corrió el modelo original por ciudad, es decir se tomaron los datos de cada ciudad por separado y se realizó el logit ordenado en Bogotá, Buenaventura, Cali y Cartagena. A continuación se presentan las ciudades que son representativas. Estas son Cali y Cartagena como se puede apreciar en las tablas 10 y 11.

En Cali, se encontró un r2 del 40\%; así mismo, en Buenaventura, las variables experiencia, número de integrantes de la organización y el haber sido finalistas tienen una relación negativa con la innovación social. En Bogotá, tanto la experiencia como los integrantes de la organización poseen relaciones inversas con la variable dependiente, al igual que en Cali.

Observando las salidas de forma independiente, se concluye que en ninguna de las ciudades hay variables significativas al 95\% de confianza, sin embargo en Cartagena se cumplen todas las hipótesis propuestas en un principio y es donde se explica en mayor porcentaje el comportamiento de la innovación social, alcanzando un $\mathrm{r} 2$ de 0,77 , también en esta ciudad la variable 
Tabla 9

Innovación, participantes, finalista, financiamiento

$\begin{array}{lll}\text { Iterration 0: } & \text { log likelihood }=-79,281319 \\ \text { Iterration 1: } & \log \text { likelihood }=-75,767363 \\ \text { Iterration 2: } & \log \text { likelihood }=-75,662236 \\ \text { Iterration 3: } & \log \text { likelihood }=-75,661866 \\ \text { Iterration 4: } & \log \text { likelihood }=-75,661866\end{array}$

Ordered logistic regression

Number of obs - $\quad-135$

Log likelihood - - 75,661836

LR chi2(4) - $\quad-7,24$

Prob $>$ chi2 $\quad-\quad-0,1238$

\begin{tabular}{lccccccc} 
& & & & & Pseudo R2 & $-0,0457$ \\
\hline Innovación & Coef. & Std. Err. & $\mathbf{z}$ & $\mathrm{P}>|\mathbf{z}|$ & \multicolumn{2}{c}{ [95\% Cont. Interval] } \\
Participantes & 0,0007038 & 0,000642 & 1,10 & 0,273 & $-0,0005546$ & 0,0019621 \\
Ha sido finalista & 0,7020658 & 0,5335276 & 1,32 & 0,188 & $-0,3436292$ & 1,747761 \\
Financiamiento & 0,0552152 & 0,0478802 & 1,15 & 0,249 & $-0,0386282$ & 0,1490585 \\
Personería & 0,6228734 & 0,4604004 & 1,35 & 0,176 & $-0,2496123$ & 1,576807 \\
\hline \multicolumn{1}{c}{$/$ cut1 } & 2,4679958 & 0,8529996 & & & 0,2794949 & 4,139806 \\
\hline
\end{tabular}

Nota. Elaboración propia.

Tabla 10

Logit para Cali

Iterration 0 :

Iterration 1:

Iterration 2:

Iterration 3:

Iterration 4:

Iterration 5:

Iterration 6:

Iterration 7:

Iterration 8:

Iterration 9:

Iterration 10:

$\log$ likelihood $=-15,330972$

$\log$ likelihood $=-12,210848$

$\log$ likelihood $=-10,149212$

log likelihood $=-9,4026961$

$\log$ likelihood $=-9,169047$

log likelihood $=-9,1081235$

log likelihood $=-9,105444$

$\log$ likelihood $=-9,1048187$

$\log$ likelihood $=-9,1046889$

$\log$ likelihood $=-9,1046656$

$\log$ likelihood $=-9,1046632$

Iterration 11: $\quad \log$ likelihood $=-9,1046627$

Ordered logistic regression

Number of obs - $\quad 42$

Log likelihood - -9,1046627

LR chi2(4) _ - 12,45

Prob > chi2 - 0,0526

Pseudo R2 _ _ $\quad 0,4061$

\begin{tabular}{lcccccc}
\hline Innovación & Coef. & Std. Err. & $\mathbf{z}$ & $\mathbf{P}>|\mathbf{z}|$ & \multicolumn{2}{c}{ [95\% Cont. Interval] } \\
\hline Experiencia & $-0,0317192$ & 0,169201 & $-0,19$ & 0,579 & $-0,3633472$ & 0,2999087 \\
Integrantes & $-0,0709206$ & 0,0804592 & $-0,88$ & 0,602 & $-0,2286176$ & 0,0867765 \\
Participantes & 0,0037122 & 0,0023302 & 1,59 & 0,468 & $-0,000855$ & 0,0082794 \\
Ha sido finalista & 0,584592 & 1,319243 & 0,44 & 0,199 & $-2,001077$ & 3,170264 \\
Financiamiento & 0,6515911 & 0,4724679 & 1,38 & 0,249 & $-0,275017$ & 1,578199 \\
Personería & 16,47794 & 3053,189 & 0,01 & 0,996 & $-5967,64$ & 6000,596 \\
\hline \multicolumn{1}{c}{ /cut1 } & 2,388848 & 0,8863451 & & & $-5955,404$ & 6012,878 \\
\hline
\end{tabular}

Nota. Elaboración propia. 
Tabla 11

Logit para Cartagena

Iterration 0:

$\log$ likelihood $=-17,148245$

Iterration 1:

$\log$ likelihood $=-5,1096121$

Iterration 2:

$\log$ likelihood $=-4,1897825$

Iterration 3:

Iterration 4:

$\log$ likelihood $=-3,8396157$

Iterration 5:

$\log$ likelihood $=-3,8196157$

Iterration 6:

$\log$ likelihood $=-3,819423$

$\log$ likelihood $=-3,8194228$

Ordered logistic regression

Number of obs -

Log likelihood - - -3,8194228

LR chi2(6) _ - 26,66

Prob $>$ chi2 - 0,0002

\begin{tabular}{lcccccc}
\hline Innovación & Coef. & Std. Err. & $\mathbf{z}$ & $\mathrm{P}>|\mathbf{z}|$ & \multicolumn{2}{c}{ [95\% Cont. Interval] } \\
\hline Experiencia & 1,207119 & 0,6571537 & 1,84 & 0,066 & $-0,0808789$ & 2,495116 \\
Integrantes & 0,0045481 & 0,0126539 & 0,36 & 0,719 & $-0,0202531$ & 0,0293492 \\
Participantes & $-0,1410325$ & 0,1302005 & 1,08 & 0,279 & $-0,1141558$ & 0,3962209 \\
Ha sido finalista & 6,654875 & 17,49021 & 0,38 & 0,704 & $-27,62531$ & 40,93505 \\
Financiamiento & $-0,6314513$ & $-0,7234265$ & 0,87 & 0,383 & $-0,7864387$ & 2,049341 \\
Personería & 9,02558 & 6,937492 & 1,30 & 0,193 & $-4,571655$ & 22,62281 \\
\hline \multicolumn{1}{c}{$/$ cut1 } & 29,72043 & 21,12515 & & & $-11,6841$ & 71,12497 \\
\hline
\end{tabular}

Nota. Elaboración propia.

experiencia alcanza a ser significativa al 90\% de confiabilidad, y el modelo en su conjunto es significativo, de acuerdo con el test de Wald, es menor a 0,05.

\section{Resultados del estudio cualitativo}

En el apartado del estudio cualitativo se presentan los resultados tanto en redes semánticas como en nubes de palabras.

En la figura 5, se pueden apreciar las principales áreas de trabajo de las iniciativas: actividades culturales, actividades en torno a la salud, especialmente lo que tiene que ver con la nutrición, trabajo en medio ambiente, el desarrollo comunitario, actividades deportivas entre las cuales se destacan el fútbol, patinaje, tenis. Otras áreas de trabajo de estas iniciativas tienen que ver con los servicios terapéuticos, el consumo de drogas y temas de familia.

En cuanto a la variable "Edad" (figura 6), se encuentran grupos etáreos desde los 7 hasta los 85 años, esto tiene relación con la anterior red analizada (figura 5), ya que se evidencia el amplio portafolio de servicios que tienen las iniciativas que participaron, por lo cual la oferta debe ser tan amplia que considere los diferentes segmentos de edad que se encuentran.

Asimismo, en la figura 7, los principales elementos de la misión que se encuentran en el presente análisis de organizaciones e iniciativas están enmarcados en el mejoramiento de la calidad de vida, la construcción de paz, la promoción de la cultura, los espacios de participación, el aprovechamiento del tiempo libre, el fortalecimiento del tejido social.

Dicho de otro modo, en la misión de las organizaciones, se resalta la importancia del desarrollo comunitario mediante una formación integral de la población, procurando la construcción de proyectos de vida principalmente mediante iniciativas culturales y ambientales. El tamaño de las palabras desarrollo, comunidad, integral es relevante ya que en sus misiones parten de la búsqueda del impacto en la comunidad con la cual trabajan. Se puede apreciar esto mismo con las palabras jóvenes, personas y calidad, que reafirman la búsqueda por contribuir con el mejoramiento de condiciones de vida.

De modo similar, en la figura 8 , se resalta el foco de las iniciativas hacia los jóvenes, niños y niñas mediante formación en diversos ámbitos que permitan la generación de valores, el empleo del tiempo libre y el desarrollo tanto a nivel personal como comunitario. Se destaca el carácter urbano de la mayor parte de las iniciativas. Nuevamente, aparece la palabra vida, que da cuenta de elementos que aportan a la construcción de aspectos que mejoren las condiciones de esta, y de formación y de actividades que impacten este aspecto. 


\section{Áreas de trabajo}

\begin{tabular}{|l|}
\multicolumn{1}{|c|}{ Eventos culturales } \\
\hline Pintura \\
\hline Baile \\
\hline Comunicación \\
\hline Educación musical \\
\hline Arte y cultura \\
\hline Labor artística \\
\hline Música \\
\hline \\
\hline Cemprendimiento laboral \\
\hline Manualidades \\
\hline Costura \\
\hline
\end{tabular}

\begin{tabular}{|l|}
\hline \multicolumn{1}{|c|}{ Salud } \\
\hline Nutrición \\
\hline Desayunos para niños \\
\hline Valoración nutricional \\
\hline Ambientes saludables \\
\hline
\end{tabular}

\begin{tabular}{|l|}
\multicolumn{2}{|c|}{ Servicios terapéuticos } \\
\hline Bienestar psicosocial \\
\hline Consumo de drogas \\
\hline Violencia intrafamiliar \\
\hline Familia \\
\hline
\end{tabular}

\begin{tabular}{|l|}
\multicolumn{1}{|c|}{ Problemática social } \\
\hline Promoción de derechos humanos \\
\hline Grupos de defensa civil \\
\hline Cuidado del medio ambiente \\
\hline Autonomía y capacidad de aporte \\
\hline Comunidad \\
\hline Medio ambiente \\
\hline $\begin{array}{l}\text { Capacitaciones de sensibilización a la } \\
\text { comunidad }\end{array}$ \\
\hline
\end{tabular}

\begin{tabular}{|l|}
\hline \multicolumn{1}{|c|}{ Deporte } \\
\hline Fútbol \\
\hline Defensa personal \\
\hline Beisbol, tenis, patinaje, hockey \\
\hline Ultimate \\
\hline
\end{tabular}

\section{Procesos pedagógicos}

Formación complementaria en contabilidad, administración, servicio al cliente, mercadeo

Jornadas extracurriculares de aprovechamiento del tiempo libre para niños

Capacitaciones sobre discapacidad

Educación especial

Capacitación a los padres de familia

Investigación

Educación popular

Centro especializado de aprendizaje para niños y niñas

Figura 5. Áreas de Trabajo. Elaboración propia.

\section{Edades de los beneficiarios}

\begin{tabular}{l} 
Niños entre los 7 - 12 años \\
11 años \\
14 años en adelante \\
5 años hasta los 12 años \\
6 y 15 años de edad \\
4 - 14 años \\
Niños desde los 6 años \\
12 a 5 años \\
5 y los 12 años \\
Menores entre 0 y 12 años \\
Niños de 0 a 12 años \\
Adolescentes entre 12 y 17 años \\
1 a 10 años \\
\hline
\end{tabular}

\begin{tabular}{|l}
\hline 15 - 19 años \\
14 y 30 años \\
\hline 30 a 65 años \\
13 años hasta los 22 años \\
Mujeres mayores de 30 años \\
14 y 27 años \\
\hline
\end{tabular}

Madres de familia

Madres gestantes e hijos

hasta los 3 años
6 a 20 años

[1:35] Jóvenes entre los 13 y 21 años

[1:42] 15 a 20 años

[1:53] 18 años

[1:25] 15 y 60 años

[1:33] Adulto mayor entre los 50 - 85 años

[1:32] Más de 60 años

[1:39] Más de 60 años

Figura 6. Edades. Elaboración propia. 
Contribuir al mejoramiento de la calidad de vida de los niños

Mejorar la calidad de vida de los niños y niñas, adolescentes y adultos de escasos recursos en condición de vulnerabilidad, del corregimiento de la buitrera

Mejorar la calidad de vida de nuestras comunidades especialmente la de los niños y niñas, sin olvidar su núcleo familiar

Satisfacer la necesidad de los niños, niñas, mujeres y familias con estándares de calidad

Ofrecer y crear el espacio idóneo para el desarrollo del ser

Promover y fortalecer en el eindividuo, el sentido de pertenencia frente a su comunidad, localidad y el Distrito Capital a través del ejercicio de la democracia participativa y desarrollar procesos para la recuperación, recreación y fomento de las diferentes manifestaciones culturales, recreativas y deportivas, que fortalezcan la identidad comunal

Busca la formación cultural e intelectual de la población de la localidad de San Cristobal

Promueve en la humanidad cultura sobre gestión del riesgo de desastres y desarrollo sostenible, en busca de la apropiación del nuevo conocimiento
Contribuir con la construcción de paz y convivencia en Colombia y en el mundo, promoviendo cultura, pedagogía y espiritualidad del cuidado, el perdón y la reconciliación

Propiciar una sala convivencia social en el ámbito familiar, escolar, comunitario

Fortalecer el tejido social a través de las manifestaciones culturales

Contribuir a la construcción de procesos sociales

Construir capital humano y social

Contribuir a la formación y desarrollo integral de la población

Propender por el desarrollo de las personas que lo integran: mediante la disciplina, El deseo de los logros, la perseverancia, en entrenamientos

Por medio de la práctica del fútbol, los valores en niños y jóvenes, el cuidado del medio ambiente, respeto por la vida

Aprovechamiento del tiempo libre en formación cultural y deportiva

La bicicleta educativa es un proyecto institucional que busca generar en los niños, niñas y en la comunidad educativa en general el colegio Brasilia Bosa, conocimiento y apropiación de su localidad, formación ciudadana, educación vial y ambiental, por medio de la utilización de un medio de transporte sotenible como lo es la bicicleta
Brindar procesos de rehabilitación y educación integral de calidad según las habilidades y capacidades de los niños, niñas y jóvenes en situación de discapacidad

Promueve espacios de participación y compromiso que involucre y beneficie a niños y niñas, jóvenes y adultos hacia la paz y la construcción de la misma

Desarrollará, promoverá y ejecutará programas y proyectos, en materia de educación, salud, vivienda, derechos humanos, medio ambiente, cultura, alimentación, recreracion y deporte

Psicosocial de los niñosy niñas basado en la construcción y fortalecimiento del vínculo afectivo madre - hijo, mediante el acompañamiento efectivo, desde la gestacion hasta los tres años de vida del niño

Separación de la fuente y fomento del reciclaje que permita la transformación y comercialización del material, y que a su vez se convierta en fuente de empleo y generación de ingresos

Convertir a Cali en un centro reconocido a nivel nacional de desarrollo del clown

Figura 7. Elementos de la misión. Elaboración propia.

Respecto a las respuestas en torno al contexto donde se desarrolla la iniciativa, como se puede apreciar en la figura 9, la población juvenil es la más sobresaliente dentro de la comunidad. Los problemas más reiterativos en el territorio son el deterioro ambiental, la pobreza y los fenómenos de violencia, esta violencia según la descripción detallada de diversas iniciativas se da a distintos niveles, como violencia intrafamiliar, guerra de pandillas (asociada en la mayoría de ocasiones con el consumo de psicoactivos y el microtráfico); en una menor proporción, se resalta el matoneo como otra forma de violencia.

Por tanto, en las descripciones particulares de cada uno de los contextos, se observa de forma reiterativa que manifiestan la ausencia del Estado en diversas formas, consideran que no hay servicios públicos básicos, no hay condiciones de seguridad, no llega inversión del Estado, no se tiene en cuenta a la comunidad para el desarrollo de políticas, no hay servicios hospitalarios adecuados, entre otros factores mencionados.

Asimismo, en la figura 10, dentro de las problemáticas que abordan las distintas iniciativas, la más reiterativa es la falta de oportunidades, principalmente para los jóvenes, lo que se presenta en un contexto en el que la mayor parte de la población según datos arrojados en las entrevistas puede estar influenciada por aspectos sociales de inseguridad, de bandas al margen de la ley y de microtráfico. 


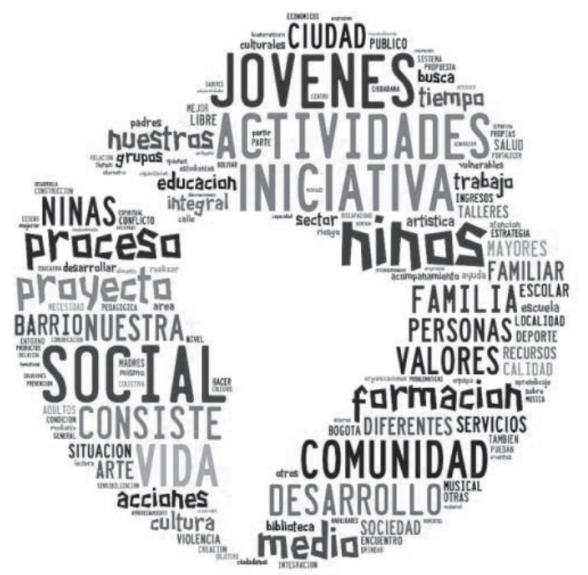

Figura 8. Nube de palabras descripción iniciativa. Elaboración propia.

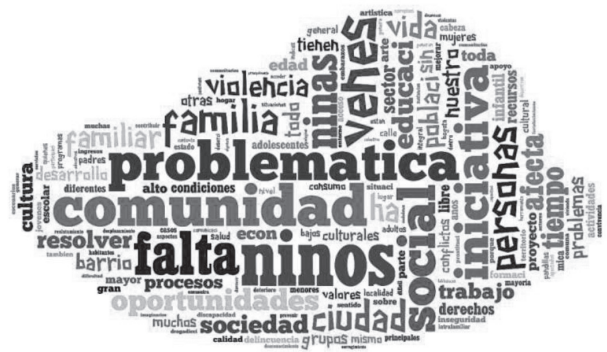

Figura 10. Nube de palabras problemática. Elaboración propia.

De otro modo, es interesante resaltar que las iniciativas reconocen la innovación social como un aspecto que permite el desarrollo de emprendimientos enmarcados dentro de un proceso comunitario que busca la generación de estrategias y alternativas a las problemáticas que se presentan en su espacio de acción. Entre los determinantes de porqué se consideran innovadoras, se resalta que la población con la que trabajan, especialmente jóvenes, niños y discapacitados permiten la integración de la familia a las iniciativas, ya que se convierte en un escenario en el cual se comparte y se puede reflexionar alrededor de las soluciones que se pueden plantear. Es innovador porque relaciona contextos inmediatos de los beneficiarios, como lo comunitario, lo familiar, lo escolar, entre otros (figura 11).

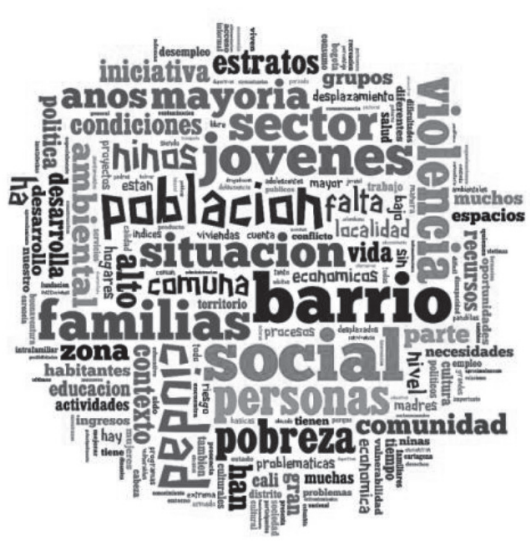

Figura 9. Nube de palabras contexto. Elaboración propia.

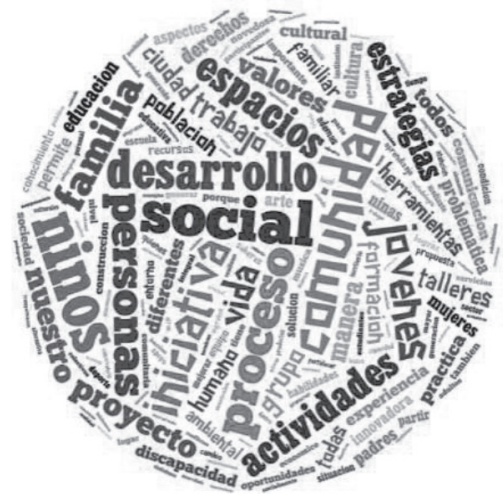

Figura 11. Nube de palabras innovación social. Elaboración propia.

Como se puede apreciar en la figura 12, entre los cambios duraderos, se resalta de nuevo a los jóvenes y niños como la comunidad más afectada, en donde los principales cambios se representan en un mejoramiento en las condiciones de vida, aumento de la participación en los procesos sociales, mejoramiento en las relaciones familiares del barrio a través de la generación de nuevos espacios de trabajo en el territorio.

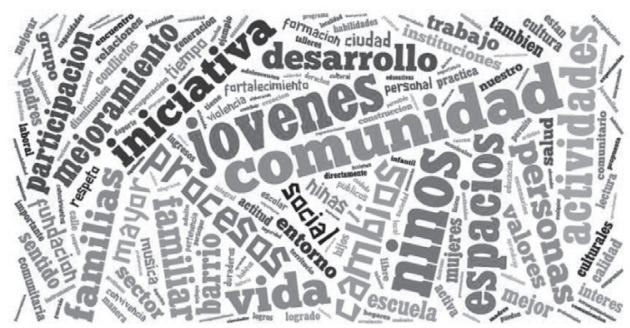

Figura 12. Nube de palabras cambios duraderos. Elaboración propia. 
Respecto de los principales riesgos que puedan amenazar la sostenibilidad del proyecto (figura 13), está la falta de recursos de todo tipo, pero principalmente económicos; esto es reiterativo prácticamente en todas las iniciativas. Por otro lado, otros riesgos recurrentes son la falta de espacio para el desarrollo de actividades y la dificultad de conseguir nuevos participantes en los proyectos. Se plantea como un desafío involucrar a las familias de los directos beneficiarios de los proyectos, principalmente en los casos en los que se trabaja con población discapacitada.

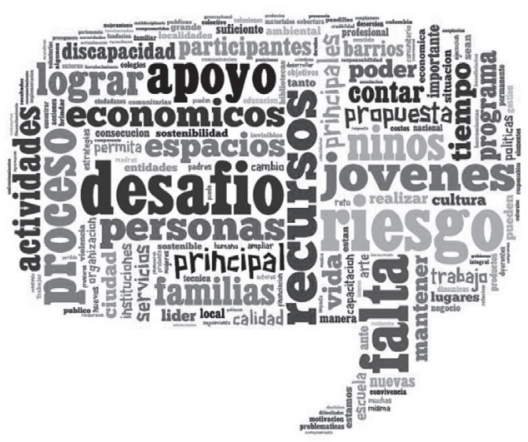

Figura 13. Nube de palabras riesgos. Elaboración propia.

Como se puede apreciar en la figura 14, en relación con el futuro (dos años) que identifican los integrantes de las iniciativas, se evidencian las intenciones de escalarlas a mayores niveles, sacarlas del barrio, ampliar su cobertura, para que estas lleguen a niveles locales, nacionales y en menor medida a niveles internacionales; para esto identifican como un factor clave la cooperación con otros actores con los que actualmente muchas no están involucradas fuertemente, estás son las instituciones del Estado y el sector empresarial. Por otro lado, las iniciativas se interesan cada vez más en desarrollar sus estructuras organizativas y sus procesos internos en aras de tener organizaciones más maduras.

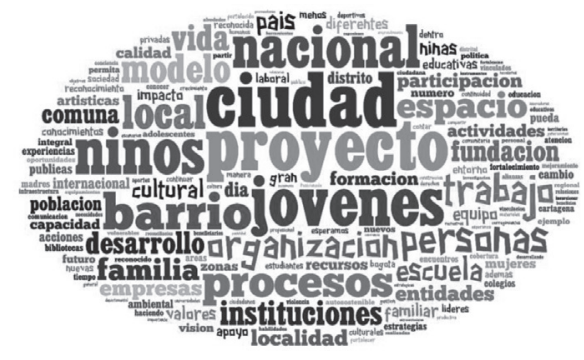

Figura 14. Nube de palabras futuro. Elaboración propia.

\section{Conclusiones}

Durante las 15 versiones del premio, se evidencia la capacidad que tienen las organizaciones para realizar el trabajo con las comunidades a partir de situaciones identificadas, permitiéndole a algunas de estas la participación en escenarios públicos y en temáticas como la educación, la pobreza, la desigualdad, el ambiente, entre otros.

La innovación social se convierte en una posibilidad que tienen las organizaciones, para proyectarse en la posible solución a problemáticas que se presentan en su entorno inmediato, con el presente estudio se identifica como elementos que efectivamente se encuentran en las iniciativas analizadas, las ideas + propuestas surgen de la misma comunidad, en muchas de ellas son soluciones a pequeña escala, que buscan prototiparse para replicarse en otros entornos en condiciones similares.

Las iniciativas que se presentaron en la versión del 2013, le apuestan a contribuir al mejoramiento de condiciones de vida de su espacio local.

Por el tamaño de estas, no es tan fácil acceder y articular el trabajo con políticas públicas, ya que se dedican al día a día y a procurar participar con sus comunidades inmediatas. Otra dificultad que se presenta está enmarcada dentro de la sostenibilidad, ya que son ideas interesantes y que procuran mejores condiciones de vida, pero que afrontan problemas para generar liquidez en el tiempo.

Es importante realizar el proceso de fortalecimiento a la luz de elementos organizacionales, es decir, en el manejo presupuestal, en la formulación y presentación de proyectos, en el desarrollo de fondeo de recursos (fundraising), en el acercamiento, la apropiación y el diseño de alianzas con otras organizaciones y actores claves que permitan darle solidez a los procesos.

Las iniciativas procuran el fortalecimiento de procesos de educación y de apropiación de elementos culturales de los entornos en los cuales se desarrollan, por esta razón es importante que se sigan apoyando en la generación de redes con diversos actores que les permitan visibilizarse mucho más a nivel local y nacional.

Gracias a que algunos de los líderes de las iniciativas toman como un proyecto de vida su participación, se puede generar la continuidad, pero esto se puede convertir en algún momento en un riesgo ya que se debe trabajar en el empoderamiento de las comunidades, puesto que si el líder del proceso se agota 
o siente que no puede continuar, las iniciativas pueden desaparecer.

El país no está preparado para la recolección de datos que evidencien o expliquen el fenómeno de la innovación social, por esta razón al realizar modelos en este sentido se encuentran vacíos en los criterios de validación, tanto en la significancia individual como en el modelo en conjunto.

La dinámica de la innovación social es diferente en cada territorio, es decir, no existe uniformidad en el criterio, lo que ocasiona resultados muy diferentes entre ciudades, esto puede ser un punto de partida para orientar la política pública de innovación social que conduzca a una identidad nacional.

Es evidente la pertinencia de crear una encuesta de innovación social por parte del sector público, que recoja la información de todas las organizaciones sociales que se encuentran trabajando en el país, de esta podría derivarse un modelo transversal a cualquier organización o iniciativas.

\section{Referencias}

Alcaldía Buenaventura. (2014). Recuperado de http:// www.buenaventura.gov.co/secciones/2/1/1914/comunas-de-buenaventura

Alter, S. (2002). Case studies in social entrepreneurship. Washington D.C.: Counterpart International.

Bouchard, M. (2007). Los escenarios nacionales Québec, Canada: la economía social en Quebec: protagonista de la innovación y de las transformaciones sociales. En V. Mirta (ed.), La co-construcción de políticas públicas en el campo de la economía social. Buenos Aires: Prometeo Libros.

Davidson, R. y MacKinnon, J. (2004). Discrete and limited dependent variables. En Econometric Theory and Methods (pp. 451-475). New York: Oxford University Press.
Escobar-Delgado, R. A. (2010). Las ONG como organizaciones sociales y agentes de transformación de la realidad: desarrollo histórico, evolución y clasificación. Diálogo de saberes investigaciones en derecho y ciencias sociales, 32, 121-132. Recuperado de http://www.unilibre.edu. co/dialogos/admin/upload/uploads/Articulo\%208. pdf

Etzioni, A. (1975). Organizaciones modernas. México: Uteha, Editorial Plaza y Janés.

Francke, M. y Morgan, M. D. (1995). La sistematización: apuesta por la generación a partir de las experiencias de promoción. Materiales Didácticos, 1. Recuperado de http://www.alboan.org/archivos/343.pdf

Hollingsworth, R. J. (2000). Doing institutional analysis. Review of International Political Economy, 7 (4), 595-644.

Observatorio Social de Cali (2014). Perfiles por comunas. Recuperado de http://www.cali.gov.co/ipc/publicaciones/perfiles_por_comunas_pub

Pérez, G. J. y Salazar-Mejía, I. (2007). La pobreza en Cartagena un análisis por barrios. Recuperado de http:// www.banrep.gov.co/docum/ Lectura_finanzas/pdf/ DTSER-94.pdf

Premio Cívico (2013). Premio Cívico por una Ciudad Mejor la Innovación Social Transforma la Ciudad. Recuperado de http://www.porunaciudadmejor.com/informacion/el-premio

Richard, H. (1983). Organizaciones: estructura y proceso. México: Prentice Hall.

Rocha, D. (agosto del 2011). Parque científico de innovación social (Documento de trabajo). Uniminuto, Bogotá, Colombia.

Scott, J. F. (1963). The Changing Foundations of the Parsonian Action Schema. American Sociological Review, 28, 716-735. Recuperado de http://www.jstor.org/ stable/2089910?seq=1\#page_scan_tab_contents. doi http://dx.doi.org/ 10.2307/ 2089910 